\title{
Nutrient deficiencies in patients with functional bowel disorders
}

\author{
M. Szuba ${ }^{1}$, P. Stribling ${ }^{1}$, C. Ball ${ }^{1}$ and F. Ibrahim ${ }^{1}$ \\ ${ }^{1}$ Life Sciences Directorate, University of Suffolk, Ipswich, Suffolk, UK
}

People suffering from Functional Bowel Disorders (FBDs) often associate their symptoms with the consumption of specific foods. Thus, dietary adjustments are often implemented to alleviate the symptoms of FBDs, and this commonly involves the exclusion of certain food groups ${ }^{(1-2)}$. This is generally achieved by following the low FODMAP diet, gluten-free diet, or various other online dietary plans, which are often implemented without professional advice ${ }^{(3-4)}$. This study aimed to evaluate whether self-prescribed dietary modifications may put those with FBDs at risk of nutritional deficiencies in the long term.

Thirty-seven participants took part in the current study to assess their dietary nutrient intakes. The participant selection criteria included men and women aged 18 years or over, suffering from any form of FBD. The participants were recruited using email, social media, online forums, flyers, and bulletin boards in shops, as well as sports and community centres across the Suffolk region, UK. All participants gave informed consent, and the study was approved by the University of Suffolk Ethics Committee. The participants filled in a food frequency questionnaire (FFQ), and the FFQ EPIC Tool for Analysis (FETA) software was used to convert food frequencies into separate nutrient intakes.

Each estimated average nutrient intake was assessed against the respective Reference Nutrient Intake (RNI), set by SACN/COMA (1991), using the one-sample t-test (IBM SPSS Statistics 25).

When compared with the reference nutrient intakes (RNIs) for each nutrient, the estimated participant nutrient intakes were significantly lower $(\mathrm{p}<0.05)$ for vitamin $\mathrm{D}$, magnesium, iodine, iron, selenium, potassium, and non-starch polysaccharides, and they were adequate $(p>0.05)$ for copper, folate, and calcium. Nutrient intakes were significantly higher $(p<0.05)$ for vitamins B1, B2, B3, B6, $\mathrm{B} 12, \mathrm{~A}, \mathrm{C}$, and $\mathrm{E}$, manganese, sodium, zinc, chloride, and phosphorus, but none of these nutrients reached any established upper tolerable levels. The intakes of total energy and free sugars were close to the recommended values, whereas the intakes of total carbohydrates, saturated fat, and total fat exceeded the recommended values (SACN 2015).

As the majority of participants had an inadequate intake of certain micronutrients, those with FBDs should seek professional advice before the implementation of dietary changes. However, due to the inherent limitation associated with FFQs, further research using a 7-day food record is warranted to confirm the current findings.

\section{References}

1. Nwosu BU, Maranda L \& Candela N (2017) PLoS One 12, e0172183.

2. Oka P, Parr H, Barberio, et al. (2020) The Lancet Gastroenterol 5, 908-917.

3. Okawa Y, Fukudo S \& Sanada H (2019) BioPsychoSocial Medicine 13.

4. O'Keeffe M, Jansen C, Martin, et al. (2021) Neurogastroenterol and Motil 30, e13154. 